\title{
Mathematical Analysis of Deterministic and Stochastic Model of Tuberculosis
}

\author{
Texance MBAYA ${ }^{\dagger}$, Jean Luc DIMI ${ }^{\dagger},^{\dagger} *$, Bienvenu ONDAMI \\ † Département de Mathématiques, Université Marien Ngouabi, \\ BP 69 Brazzaville Congo \\ ‡ Institut National de la Recherche en Sciences Exactes et Naturelles \\ Avenue de l'Auberge de Gascogne \\ BP 2400 Brazzaville Congo \\ Corresponding Author Email Address: jldimi@yahoo.fr
}

\begin{abstract}
The main purpose of this paper is to explore the deterministic and stochastic model of tuberculosis. The existence and stability of equilibria are analysed.We also give an equivalent stochastic equation model for simulations.
\end{abstract}

Keywords: Nonlinear epidemic model; Lyapounov function; stochastic asymptotic stability; Itô's formula

\section{INTRODUCTION}

In recent years the mathematical modeling of infectious diseases is well described in the litterature. Diseases that are transmitted directly from person to person are modelled by using the SEIR system.

Tuberculosis (TB) is an infection disease caused by Mycobacterium tuberculosis, which is transmitted from an infected person to a susceptible person in airborne particles, called droplet nuclei. These are 1 to 5 microns in diameter. These infectious droplet nuclei are tiny water droplets with the bacteria that are released when persons who have pulmo- nary or laryngeal tuberculosis cough, sneeze, laugh, shout etc. These tiny droplet nuclei remain suspended in the air for up to several hours. Tuberculosis bacteria however are transmitted through the air, not by surface contact. This means touching cannot spread the infection unless it is breathed in. Transmission occurs when a person inhales droplet nuclei containing tuberculosis bacteria. These droplet nuclei travels via mouth or nasal passages and move into the upper respiratory tract. Thereafter they reach the bronchi and ultimately to the lungs and the alveoli. According to the World Health Organization (WHO), the epidemiology of tuberculosis varies around the world, the hightest rates are observed in Sub-saharan Africa, India , Indonesia and China and are , in part, due to interactions with HIV.

In this paper we formulate and analyze dynamical and stochastic model in section 1 to 3 . The rest of the paper is organized as follows.In section 4, we derive an equivalent sto- chastic model for tuberculosis model and in section 5 computational simulations are per-formed.

\section{THE MODEL}

In this section we present the system of differential equations of tuberculosis which described the considered model of tuberculosis. We consider a given finite human po- pulation of $\mathrm{N}$ people, which we divide into three categories: susceptible, exposed and infected.

$$
\left\{\begin{array}{l}
\dot{S}=\Lambda-\beta \frac{S I}{N}-\mu S \\
\dot{E}=\beta(1-p) \frac{S I}{N}+r_{2} I-\left(\mu+k\left(1-r_{1}\right)\right) E \\
\dot{I}=\beta p \frac{S I}{N}+k\left(1-r_{1}\right) E-\left(\mu+d+\delta+r_{2}\right) I
\end{array}\right.
$$


$\Lambda$ is the recruitment into the population ; $\beta$, the probability that a susceptible individual will be infected by infectious ; $\mu$ is the probability that an individual in the population died from reasons not related to the desease; $d$ is the probability that an infectious individual dies because of the desease.An individual leaves his region to another for a new treatment with the probability $\delta$, thus this individual goes missing of model. To account for treat- ment, we define $\mathrm{r}_{1} \mathrm{E}$ as the fraction of population receiving effective chemosprophyilaxis and $r_{2}$ as the rate of effective per capita therapy. We assume that chemosprophylaxis of latently infected individuals $\mathrm{E}$ reduces their reactivation rate $r_{1}$ and that the initiation of therapeutics immediatly removes individuals from active status $\mathrm{I}$ and places them into state $\mathrm{E}$, the time before latently infected individuals who does not received effective che- moprophylaxis become infectious is assumed to satisfy an exponential distribution, with time $\frac{1}{k}$. Thus, individuals leave the class $\mathrm{E}$ to $\mathrm{I}$ at rate $\mathrm{k}\left(1-\mathrm{r}_{1}\right)$. Also, after receiving a theurapeutic treatment, individuals leave the class I to $\mathrm{E}$ at rate $\mathrm{r}_{2} \mathrm{I}$. By adding the System (1), the equation for total population is given by

If there is no desease in the population

$$
\dot{N}=\Lambda-\mu N-(d+\delta) \mathrm{I}
$$

$$
\mathrm{N}=\frac{\Lambda}{\mu}
$$

The following result show that the solutions for model (1) are bounded and, hence, lie in a compact set and are continuable for all positive time.

Lemma 1: the plane $\mathrm{S}+\mathrm{E}+\mathrm{I} \leq \frac{\Lambda}{\mu}$ is an invariant manifold of model (3), which is attracting in the first octant.

Proof: Summing up the three equations, we have $N(t)=S(t)+E(t)+I(t)$. It follows from

(1) that:

$$
\dot{N}=(S+E+I)^{\prime}=\Lambda-\mu(S+E+I) \leq \Lambda-\mu
$$

Hence, by integration, we check

$$
\frac{d N}{d t} \leq \frac{\Lambda}{\mu}+\left(N(0)-\frac{\Lambda}{\mu}\right) e^{-d t}
$$

and Then :

$$
\limsup _{t \rightarrow \infty} N(t) \leq \frac{\Lambda}{\mu}
$$

So the feasible region for system (1) :

$$
\Delta=\left\{(S, E, I) \in \mathbb{R}_{+}^{3}, 0 \leq(S+E+I) \leq \frac{\Lambda}{\mu}\right\}
$$

is positively invariant.Therefore, for initial starting point $\mathrm{x} \in \mathbb{R}_{+}^{3}$, the trajectory lies in $\stackrel{\circ}{\Delta}$

Theorem 1 :

System (1) does not have nontrivial periodic orbits.

Proof: From lemma 1, we can say that a limit cycle, if it exists, must lies in the region

Consider system (1) for $\mathrm{S}>0$ and $\mathbf{I}>0$. Take a Dulac function

We have

$$
\mathrm{D}(\mathrm{S}, \mathrm{I})=\frac{\beta}{S I}
$$

$$
\frac{\partial(D P)}{\partial S}+\frac{\partial(D Q)}{\partial I}=-\frac{\beta \Lambda}{S^{2} I}-\frac{\beta}{S I^{2}} k\left(1-r_{1}\right) E<0
$$

Hence (1) does not have a limit cycle in (1).

The conclusion follows. 


\subsection{Equilibria and basic reproduction number}

System (1) has two equilibrium points: the desease equilibrium $E_{1}=\left(\frac{\Lambda}{\mu}, 0,0\right)$ and the endemic equilibrium:

$$
\mathbf{E}_{2}\left(S^{*}, E^{*}, I^{*}\right)=\left\{\begin{array}{l}
S^{*}=\frac{\Lambda\left[\beta-(d+\delta) R_{0}\right]}{\mu(\beta-d-\delta)] R_{0}} \\
E^{*}=\frac{\Lambda\left(R_{0}-1\right)\left[\beta(1-p)+r_{2} R_{0}\right]}{\left[\mu+k\left(1-r_{1}\right)\right](\beta-d-\delta) R_{0}} \\
I^{*}=\frac{\Lambda\left(R_{0}-1\right)}{\beta-d-\delta}
\end{array}\right.
$$

satisfying the system :

$$
\left\{\begin{array}{l}
\Lambda-\beta \frac{S I}{N}-\mu S=0 \\
\beta(1-p) \frac{S I}{N}+r_{2} I-\left(\mu+k\left(1-r_{1}\right)\right) E=0 \\
\beta p \frac{S I}{N}+k\left(1-r_{1}\right) E-\left(\mu+d+\delta+r_{2}\right) I=0 \\
\Lambda-\mu N^{*}-\left(d+\delta^{*}\right) I^{*}=0
\end{array}\right.
$$

Proposition: The basic reproduction number is

$$
\mathcal{R}_{0}=\frac{\left.\beta\left[\mu p+k\left(1-r_{1}\right)\right]+k\left(1-r_{1}\right)\right)+\mu r_{2}}{(\mu+d+\delta)\left(\mu+k\left(1-r_{1}\right)\right)+\mu r_{2}}
$$

Proof: The basic reproduction $\mathrm{R}_{0}$ will be calculated by using the next generation matrix from Driessche and Woutmough, 2002 [8].

Let $\mathrm{X}=(\mathrm{E}, \mathrm{I}, \mathrm{S})$. system (1) can be written as $\frac{d X}{d t}=\mathcal{F}_{\mathrm{t}}-v$, where

$$
\mathcal{F}=\left(\begin{array}{l}
\beta(1-p) \frac{S I}{N} \\
\beta p \frac{S I}{N} \\
0
\end{array}\right) \text { and } \mathcal{V}=\left(\begin{array}{l}
-r_{2} I+\left(\mu+k\left(1-r_{1}\right) E\right. \\
-k\left(1-r_{1}\right) E+\left(\mu+d+\delta+r_{2}\right) I \\
-\Lambda+\beta \frac{S I}{N}+\mu S
\end{array}\right)
$$

The jacobian matrices of $\mathcal{F}$ and $v$ at the desease free equilibrium $X_{0}$ are respectively: Where

$$
\begin{gathered}
\mathcal{D} \mathcal{F}\left(X_{0}\right)=\left(\begin{array}{ll}
F & 0 \\
0 & 0
\end{array}\right) \text {. and } \mathcal{D} \mathcal{V}\left(X_{0}\right)=\left(\begin{array}{cc}
V & 0 \\
J_{1} & J_{2}
\end{array}\right) . \\
F=\left(\begin{array}{ll}
0 & 0 \\
\beta(1-p) & \beta p
\end{array}\right) \text { and } V=\left(\begin{array}{ll}
\mu+k\left(1-r_{1}\right) & -k\left(1-r_{1}\right) \\
-r_{2} & \mu+d+\delta+r_{2}
\end{array}\right) . \\
F V^{-1}\left(\begin{array}{lll}
0 & 0 \\
\frac{\beta(1-p) k\left(1-r_{1}\right)+\beta p\left(\mu+d+\delta+r_{2}\right)}{(\mu+d+\delta)\left(\mu+k\left(1-r_{1}\right)\right)+\mu r_{2}} & \frac{\left.\beta[\mu p+k(1-r 1)]+k\left(1-r_{1}\right)\right)+\mu r_{2}}{(\mu+d+\delta)\left(\mu+k\left(1-r_{1}\right)\right)+\mu r_{2}}
\end{array}\right) .
\end{gathered}
$$

Is the next generation matrizx of system (1)

$$
\text { The radius of } F V^{-1} \text { is } \rho\left(F V^{-1}\right)=\frac{\left.\beta\lfloor\mu p+k(1-r 1)]+k\left(1-r_{1}\right)\right)+\mu r_{2}}{(\mu+d+\delta)\left(\mu+k\left(1-r_{1}\right)\right)+\mu r_{2}}
$$

Hence, the basic reproduction number of system (1) is:

$$
\mathcal{R}_{0}=\frac{\left.\beta\left[\mu p+k\left(1-r_{1}\right)\right]+k\left(1-r_{1}\right)\right)+\mu r_{2}}{(\mu+d+\delta)\left(\mu+k\left(1-r_{1}\right)\right)+\mu r_{2}}
$$




\section{Theorem 2:}

If $R_{0}<1$, then the desease free equilibrium is globally asymptotically stable in $\Delta$

Proof: Let be the following Lyapounov function $\mathrm{V}: \Delta \rightarrow \mathbb{R}$

$$
\mathrm{V}(\mathrm{S}, \mathrm{E}, \mathrm{I})=\mathrm{I}(\mathrm{t})
$$

We have

$$
\begin{gathered}
\frac{d V}{d t}=\beta \frac{S I}{N}+\mathrm{k}\left(1-r_{1}\right) E-\left(\mu+d+\delta+r_{2}\right) I \\
=\left(\mu+d+\delta+r_{2}\right)\left(\left(\mu p+k\left(1-r_{1}\right)\right)+\mu r_{2}\right) \frac{\left(\beta p \frac{S I}{N}-\left(\left(\mu p+k\left(1-r_{1}\right)\right)+\mu r_{2}\right) I\right)}{\left(\mu+d+\delta+r_{2}\right)\left(\left(\mu p+k\left(1-r_{1}\right)\right)+\mu r_{2}\right.}+k\left(1-r_{1}\right) E
\end{gathered}
$$

Which gives us:

$$
\frac{d V}{d t} \leq\left(\mu+d+\delta+r_{2}\right)\left(\left(\mu p+k\left(1-r_{1}\right)\right)+\mu r_{2}\right)\left(\mathcal{R}_{0} S(t)-\frac{1}{\left(\mu+d+\delta+r_{2}\right)}\right) I+k\left(1-r_{1}\right) E
$$

We see that $\frac{d V}{d t} \leq 0$ for $\mathcal{R}_{0}<\frac{1}{\left(\mu+d+\delta+r_{2}\right)}<1$ and $\mathrm{E}=0$

If $\mathcal{R}_{0}<1$ then $\frac{d V}{d t}=0 \Leftrightarrow \mathrm{I}(\mathrm{t})=0$

If $\mathcal{R}_{0}=1$ then $\frac{d V}{d t}=0 \Leftrightarrow S(t)=\frac{1}{\mu+d+\delta+r_{2}}$.

Hence by LaSalle's principle[7] the disease free equilibrium is globally asymptotically stable on $\Delta$

\section{Theorem 3:}

The endemic wquilibrium of the system is globally asymptotically stable on

Proof: Let be the following Lyapounov function $\quad \mathrm{V}: \Delta \rightarrow \mathbb{R}:$

$$
V(S, E, I)=W_{1}\left[S-S^{*} \ln \frac{S}{S^{*}}\right]+W_{2}\left[I-I^{*} \ln \frac{I}{I^{*}}\right]
$$

where $W_{1}$ and $W_{2}$ are positive constant to be chosen latter. We have:

$$
\begin{gathered}
\frac{d V}{d t}=W_{1} \frac{S-S^{*}}{S}\left(\Lambda-\beta \frac{S I}{N}-\mu S\right)+W_{2} \frac{I-I^{*}}{I}\left(\beta p \frac{S I}{N}+k\left(1-r_{1}\right) E-\left(\mu+d+\delta+r_{2}\right) I\right) \\
=W_{1} \frac{S-S^{*}}{S}\left(\beta \frac{S^{*} I^{*}}{N}+\mu S^{*}-\beta \frac{S I}{N}-\mu S\right)+W_{2} \frac{I-I^{*}}{I}\left(\beta p \frac{S I}{N}+k\left(1-r_{1}\right) E-\left(\mu+d+\delta+r_{2}\right) I-\right. \\
\left.\beta p \frac{S^{*} I^{*}}{N}-k\left(1-r_{1}\right) E^{*}+\left(\mu+d+\delta+r_{2}\right) I^{*}\right) \\
\quad=W_{1} \frac{S-S^{*}}{S}\left[\beta\left(\frac{S^{*} I^{*}}{N}-\frac{S I}{N}\right)+\mu\left(S^{*}-S\right)\right] \\
+W_{2} \frac{I-I^{*}}{I}\left[\beta p\left(\frac{S I}{N}-\frac{S^{*} I^{*}}{N}\right)+k\left(1-r_{1}\right)\left(E-E^{*}\right)-\left(\mu+d+\delta+r_{2}\right)\left(I-I^{*}\right)\right] \\
=W_{1} \frac{S-S^{*}}{S}\left[\beta\left(\frac{S^{*} I^{*}}{N}-\frac{S^{*} I}{N}+\frac{S^{*} I}{N}-\frac{S I}{N}\right)+\mu\left(S^{*}-S\right)\right] \\
+W_{2} \frac{I-I^{*}}{I}\left[\beta p\left(\frac{S I}{N}-\frac{S I^{*}}{N}+\frac{S I^{*}}{N}-\frac{S^{*} I^{*}}{N}\right)+k\left(1-r_{1}\right)\left(E-E^{*}\right)-\left(\mu+d+\delta+r_{2}\right)\left(I-I^{*}\right)\right] \\
=W_{1} \frac{S-S^{*}}{S}\left[\beta\left[\frac{S^{*}}{N}\left(I^{*}-I\right)+\frac{I}{N}\left(S^{*}-S\right)\right]+\mu\left(S^{*}-S\right)\right]
\end{gathered}
$$




$$
\begin{gathered}
+W_{2} \frac{I-I^{*}}{I}\left[\beta p\left[\frac{S}{N}\left(I-I^{*}\right)+\frac{I^{*}}{N}\left(S-S^{*}\right)\right]+k\left(1-r_{1}\right)\left(E-E^{*}\right)-\left(\mu+d+\delta+r_{2}\right)\left(I-I^{*}\right)\right] \\
\left.=-W_{1} \beta \frac{I}{N} \frac{\left(S-S^{*}\right)^{2}}{S}-W_{1} \beta \frac{S^{*}}{N} \frac{\left(S-S^{*}\right)}{S}\left(I-I^{*}\right)-W_{1} \mu \frac{\left(S-S^{*}\right)^{2}}{S}\right] \\
\left.+W_{2} \beta p \frac{S}{N} \frac{\left(I-I^{*}\right)^{2}}{I}+W_{2} \beta p \frac{I^{*}}{I N}\left(S-S^{*}\right)\left(I-I^{*}\right)+\frac{W_{2}}{I} k\left(1-r_{1}\right)\left(E-E^{*}\right)\left(I-I^{*}\right)-W_{2} \frac{\left(\mu+d+\delta+r_{2}\right)}{I}\left(I-I^{*}\right)^{2}\right] \\
=-W_{1} \beta\left(\frac{I}{N}+\mu\right) \frac{\left(S-S^{*}\right)^{2}}{S}-W_{2} \frac{\left(\mu+d+\delta+r_{2}\right)}{I}\left(I-I^{*}\right)^{2}+\left(W_{2} \beta p \frac{I^{*}}{I N}-W_{1} \beta \frac{S^{*}}{S N}\right)\left(S-S^{*}\right)\left(I-I^{*}\right) \\
+\frac{W_{2}}{I} k\left(1-r_{1}\right)\left(E-E^{*}\right)\left(I-I^{*}\right)
\end{gathered}
$$

And we obtain this inequality:

$$
\begin{gathered}
\leq-W_{1} \beta\left(\frac{I}{N}+\mu\right) \frac{\left(S-S^{*}\right)^{2}}{S}-W_{2} \frac{\left(\mu+d+\delta+r_{2}\right)}{I}\left(I-I^{*}\right)^{2} \\
+\frac{\beta}{I N S}\left(W_{2}-W_{1}\right)\left[S^{*}\left(S-S^{*}\right)\left(I-I^{*}\right)^{2}+I^{*}\left(S-S^{*}\right)^{2}\left(I-I^{*}\right)\right]+\frac{W_{2}}{I} k\left(1-r_{1}\right)\left(E-E^{*}\right)\left(I-I^{*}\right)
\end{gathered}
$$

For $W_{1}=W_{2}=1$, and with the fact that is small we deduce that:

$$
\frac{d V}{d t} \diamond 0
$$

We also have:

$$
\frac{d V}{d t}=0 \quad \text { if } \quad S=S^{*} \text { and } \quad I=I^{*}
$$

Hence by LaSalle's invariance principle [7], the endemic equilibrium is globally asymptoti-cally stable on $\Delta$.

\section{STOCHASTIC MODEL}

The deterministic model and the dstochastic model have the same equilibria. Through this paper, let $\left(\Omega, F,\{F\}_{i>0}, P\right)$ be a complete espace with filtration satisfying the usual conditions (i.e. it is a right continuous and increasing while $\mathrm{F}_{0}$ contains all null sets). We define the differential operator $\mathrm{L}$ associated with 3-dimensional stochastic differential equation:

$$
\mathrm{dx}(\mathrm{t})=\mathrm{f}(\mathrm{X}(\mathrm{t})) \mathrm{dt}+\phi(\mathrm{X}(\mathrm{t})) \mathrm{dB}(\mathrm{t})
$$

Where $\phi=\left(\phi_{i}\right), i=1, ., 3$ is locally Lypchitz function.B is a three Brownian motion and

$$
f(X)=\left(\begin{array}{l}
\Lambda-\beta \frac{S I}{N}-\mu S \\
\beta(1-p) \frac{S I}{N}+r_{2} I-\left(\mu+k\left(1-r_{1}\right)\right) E \\
\beta p \frac{S I}{N}+k\left(1-r_{1}\right) E-\left(\mu+d+\delta+r_{2}\right) I
\end{array}\right)
$$

If $\mathrm{V}(\mathrm{x}, \mathrm{t})$ is a Lyapounov function, we define the action of $\mathrm{L}$ on $\mathrm{V}$ by:

$$
L V(x, t)=V_{t}(x, t)+V_{x}(x, t) f(x, t)+\frac{1}{2} \operatorname{trace}\left[\phi^{T} V_{x x}(x, t) \phi(x, t)\right]
$$

where

$$
\frac{\partial V}{\partial x}=\left(\frac{\partial V}{\partial u_{1}}, \frac{\partial V}{\partial x_{2}}, \frac{\partial V}{\partial x_{3}}\right) \text { and } \frac{\partial^{2} V}{\partial x^{2}}=\left(\frac{\partial V}{\partial x_{i} \partial x_{j}}\right) \mathrm{i}, \mathrm{j}=1,2,3
$$

with reference to Afanasev and al.[1], the following theorem holds.

\section{Theorem 4:}

Suppose that there exits a function

$$
V(t, x) \in C^{1,2}\left(\mathbb{R}, \mathbb{R}^{n}\right)
$$

and two real positive continuous functions $a$ and $b$, and a constant $K$ such that, for $|x|<K$ satisfying the inequalities 


$$
\mathrm{a}(|\mathrm{X}|) \leq \mathrm{V}(\mathrm{t}, \mathrm{X}) \leq \mathrm{b}(|\mathrm{X}|)
$$

(i) if $\operatorname{LV}(t, x) \leq 0$, then the trivial solution of (4) is stable in probability,

(ii) if there exist a continuous function $\lambda: \mathbb{R}_{+}^{0} \rightarrow \mathbb{R}_{+}^{0}$, positive on $\mathbb{R}_{+}$, such that

$$
\mathrm{LV} \leq \lambda(|x|)
$$

Then the trivial solution of (4) is asymptotically stable.

Note that for stability definition, we refer to Afanas'ev and Al.

\section{Proposition 2: (9)}

Let $g$ be locally Lipchitz function such that supp $g \subset \stackrel{\circ}{\Delta}$ Then the set

$\Delta$ is stable by (4)

Theorem 5: Assume that $\theta \leq \mu, \alpha_{2} \leq \alpha_{3}$ and $\left(\alpha_{3}-\alpha_{2}\right)\left(\mathrm{k}+r_{1}\right) \leq \alpha_{1} \mu$ holds. Then for any locally Lipchtz function $g$ such that supp $g \subset \stackrel{\circ}{\circ}$ and

$$
g_{1}^{2}=\theta_{1}\left(S-\frac{\Lambda}{\mu}\right)^{2} \text { where } \frac{\Lambda}{\mu}<\lambda
$$

the disease free equilibrium $\left(\frac{\Lambda}{\mu}, 0,0\right)$ is globally asymptotically stable.

Proof: Let $\mathrm{u}_{1}=\left(\mathrm{S}-\frac{\Lambda}{\mu}\right)$ and $\mathrm{u}_{2}=\mathrm{E}$ and $\mathrm{u}_{3}=\mathrm{I}$ and consider the Lyapounov function

$$
V_{1}=\frac{1}{2} \alpha_{1} u_{1}^{2}+\alpha_{2} u_{2}+\alpha_{3} u_{3}
$$

Where $\alpha_{i}$ are real positive constants to be chosen in the course of the proof.

$$
\begin{gathered}
f^{T} \frac{\partial V_{1}}{\partial u}=\alpha_{1}\left[-\beta \frac{\left(u_{1}+\frac{\Lambda}{\mu}\right) u_{3}}{N}-\mu u_{1}\right] u_{1}+\alpha_{2}\left[\beta(1-p) \frac{\left(u_{1}+\frac{\Lambda}{\mu}\right) u_{3}}{N}+r_{2} u_{3}-\left(\mu+k\left(1-r_{1}\right)\right) u_{2}\right] \\
\alpha_{3}\left[\beta p \frac{\left(u_{1}+\frac{\Lambda}{\mu}\right) u_{3}}{N}+k\left(1-r_{1}\right) u_{2}-\left(\mu+d+\delta+r_{2}\right) u_{3}\right] \\
=-\alpha_{1} \beta \frac{\left(u_{1}+\frac{\Lambda}{\mu}\right) u_{1} u_{3}}{N}-\alpha \mu u_{1}^{2}-\left(\alpha_{1} \mu+\left(\alpha_{2}-\alpha_{3}\right)\left(k+r_{1}\right)\right) u_{2}-\alpha_{3}\left(\mu+d+\delta+r_{2}\right) u_{3} \\
+\left(\left(\alpha_{2}+\left(\alpha_{3}-\alpha_{2}\right) p\right) \beta\right) \frac{\left(u_{1}+\frac{\Lambda}{\mu}\right) u_{3}}{N}+\alpha_{2} r_{2} u_{3}
\end{gathered}
$$

One has now

$$
\begin{gathered}
L V_{2}=f^{T} \frac{\partial V}{\partial t}+\alpha_{1} g_{1}^{2} \\
=-\alpha_{1} \beta \frac{\left(u_{1}+\frac{\Lambda}{\mu}\right) u_{1} u_{3}}{N}-\alpha_{1} \mu u_{1}^{2}-\left(\alpha_{1} \mu+\left(\alpha_{2}-\alpha_{3}\right)\left(k+r_{1}\right)\right) u_{2}-\alpha_{3}\left(\mu+d+\delta+r_{2}\right) u_{3} \\
+\left(\left(\alpha_{2}+\left(\alpha_{3}-\alpha_{2}\right) p\right) \beta\right) \frac{\left(u_{1}+\frac{\Lambda}{\mu}\right) u_{3}}{N}+\alpha_{2} r_{2} u_{3}+\alpha_{1} \theta u_{1}^{2} \\
L V_{2}=-\alpha_{1} \beta \frac{\left(u_{1}+\frac{\Lambda}{\mu}\right) u_{1} u_{3}}{N}-\left(\alpha_{1} \mu-\alpha_{1} \theta\right) u_{1}^{2}-\left(\alpha_{1} \mu+\left(\alpha_{2}-\alpha_{3}\right)\left(k+r_{1}\right)\right) u_{2}-\alpha_{3}(\mu+d+\delta) u_{3}-\left(\alpha_{3}-\alpha_{2}\right) r_{2} u_{3} \\
+\left(\left(\alpha_{2}+\left(\alpha_{3}-\alpha_{2}\right) p\right) \beta\right) \frac{\left(u_{1}+\frac{\Lambda}{\mu}\right) u_{3}}{N} \\
\leq-\alpha_{1} \beta \frac{\left(u_{1}+\frac{\Lambda}{\mu}\right) u_{1} u_{3}}{N}-\left(\alpha_{1} \mu-\alpha_{1} \theta\right) u_{1}^{2}-\left(\alpha_{1} \mu+\left(\alpha_{2}-\alpha_{3}\right)\left(k+r_{1}\right)\right) u_{2}-\alpha_{3}(\mu+d+\delta) u_{3}-\left(\alpha_{3}-\alpha_{2}\right) r_{2} u_{3}
\end{gathered}
$$

According to the preceding theorem 3 , the proof is complete. 


\subsection{Stability of endemic equilibrium}

We assume that stochastic perturbations of variables around $E_{2}=\left(S^{*}, E^{*}, I^{*}\right)$ are of white noise type, which are proportional to the respective distance of $\mathrm{S}, \mathrm{E}$ and $\mathrm{I}$ from $S^{*}, E^{*}$ and $I^{*}$. see [12].

So system (1) transformed into:

$$
\left\{\begin{array}{l}
d S=\left(\Lambda-\beta \frac{S I}{N}-\mu S\right)+\sigma_{1}\left(S-S^{*}\right) d B_{1}(t) \\
d E=\left(\beta(1-p) \frac{S I}{N}+r_{2} I-\left(\mu+k\left(1-r_{1}\right)\right) E\right) d t+\sigma_{2}\left(E-E^{*}\right) d B_{2}(t) \\
d I=\left(\beta p \frac{S I}{N}+k\left(1-r_{1}\right) E-\left(\mu+d+\delta+r_{2}\right) I\right) d t+\sigma_{3}\left(I-I^{*}\right) d B_{3}(t)
\end{array}\right.
$$

Where $\mathrm{B}_{\mathbf{i}}(\mathrm{t}) \mathrm{i}=1,2,3$ is 3-dimensional Brownian motion and $\sigma_{\mathbf{i}} \geq 0, \mathrm{i}=1,2,3$ represent the intensities of $\mathrm{B}_{\mathrm{i}}$..

The system of stochastic differentiable equation in (5) can be centered at its interior endemic equilibrium by changes:

$$
U_{1}=S-S^{*}, U_{2}=E-E^{*}, U_{3}=I-I^{*} .
$$

The linearization around this endemic equilibrium take the form:

$$
d u(t)=f(u(t)) d t+\phi(u(t)) d \xi(t)
$$

Where $\mathrm{u}(\mathrm{t})=\operatorname{col}\left(u_{1}(t), u_{2}(t), u_{3}(t)\right)$ and

$$
\begin{gathered}
\phi(u)=\left(\begin{array}{lll}
\sigma_{1} u_{1} & 0 & 0 \\
0 & \sigma_{2} u_{2} & 0 \\
0 & 0 & \sigma_{3} u_{3}
\end{array}\right) \\
J\left(E_{2}\right)=\left(\begin{array}{lll}
-\beta \frac{I^{*}}{N}-\mu & 0 & -\beta \frac{S^{*}}{N} \\
\beta(1-p) \frac{I^{*}}{N} & -\left(\mu+k\left(1-r_{1}\right)\right. & \beta(1-p) \frac{S^{*}}{N}+r_{2} \\
\beta p \frac{S^{*}}{N} & k\left(1-r_{1}\right) & \beta p \frac{S^{*}}{N}-\left(\mu+d+\delta+r_{2}\right)
\end{array}\right)
\end{gathered}
$$

and $\mathrm{f}(\mathrm{u})=\mathrm{J}\left(E_{2}\right) u(t)$. Note that The endemic equilibrium corresponds to the trivial solution of $\mathrm{u}(\mathrm{t})=0$.

Theorem 6: Assume that $\sigma_{1}^{2} \leq 2\left(\beta \frac{I^{*}}{N}+\mu\right), \sigma_{3}^{2} \leq \mu+k\left(1-r_{1}\right)$ and $\sigma_{3}^{2} \leq 2\left(\left(\mu+d+\delta+r_{2}\right)\right.$ $\left.\beta \frac{S^{*}}{N}\right)$, for any locally Lipchitz function $\mathrm{g}$ such that $\phi_{1}^{2}(S, E, I)=\sigma_{1}\left(S-S^{*}\right)^{2}, \phi_{2}^{2}(S, E, I)=$ $\sigma_{2}\left(E-E^{*}\right)^{2}$ and $\phi_{3}^{2}(S, E, I)=\sigma_{3}\left(I-I^{*}\right)^{2}$, then the endemic equilibrium is globally asymptotically stable.

Proof: Let consider a Lyapounov function $V(u)=\frac{1}{2}\left(\omega_{1} u_{1}^{2}+\omega_{2} u_{2}^{2}+\omega_{3} u_{3}^{2}\right)$ are , where $\left(\omega_{i}\right), i=1, ., 3$ are real positiuve constants to be chosen in the course of the proof.

One has:

$$
\begin{aligned}
& L V(u)=-\left(\beta \frac{I^{*}}{N}+\mu\right) \omega_{1} u_{1}^{2}-\beta \frac{S^{*}}{N} \omega_{1} u_{1} u_{3}+\beta(1-p) \frac{I^{*}}{N} \omega_{2} u_{1} u_{2}-\left(\mu+k\left(1-r_{1}\right) \omega_{2} u_{2}^{2}+\right. \\
& \left(\beta(1-p) \frac{S^{*}}{N}+r_{2}\right) \omega_{2} u_{2} u_{3}+\beta p \frac{S^{*}}{N} \omega_{3} u_{1} u_{3}+k\left(1-r_{1}\right) \omega_{3} u_{2} u_{3}+\left(\beta p \frac{S^{*}}{N}-\left(\mu+d+\delta+r_{2}\right)\right) \omega_{3} u_{3}^{2} \\
& +\frac{1}{2} \operatorname{Tr}\left[\phi^{T}(u) \frac{\partial^{2} V}{\partial u^{2}} \phi(u)\right],
\end{aligned}
$$

$$
\frac{\partial^{2} V}{\partial u^{2}}=\left(\begin{array}{lll}
\omega_{1} & 0 & 0 \\
0 & \omega_{2} & 0 \\
0 & 0 & \omega_{3}
\end{array}\right)
$$

and 


$$
\phi^{T}(u) \frac{\partial^{2} V}{\partial u^{2}} \phi(u)=\left(\begin{array}{lll}
\omega_{1} \sigma_{1}^{2} u_{1}^{2} & 0 & 0 \\
0 & \omega_{2} \sigma_{2}^{2} u_{2}^{2} & 0 \\
0 & 0 & \omega_{3} \sigma_{3}^{2} u_{3}^{2}
\end{array}\right)
$$

with

$$
\frac{1}{2} \operatorname{Tr}\left[\phi^{T}(u) \frac{\partial^{2} V}{\partial u^{2}} \phi(u)\right]=\frac{1}{2}\left[\omega_{1} \sigma_{1}^{2} u_{1}^{2}+\omega_{2} \sigma_{2}^{2} u_{2}^{2}+\omega_{3} \sigma_{3}^{2} u_{3}^{2}\right]
$$

Finally one obtains

$$
\begin{aligned}
& L V=-\left(\beta \frac{I^{*}}{N}+\mu-\frac{\sigma_{1}^{2}}{2}\right) \omega_{1} u_{1}^{2}-\beta \frac{S^{*}}{N}\left(\omega_{1}-p \omega_{3}\right) u_{1} u_{3}-\left(\mu+k\left(1-r_{1}\right)-\frac{\sigma_{2}^{2}}{2}\right) \omega_{2} u_{2}^{2} \\
& \left.-\left(\left(\mu+d+\delta+r_{2}\right)-\beta p \frac{S^{*}}{N}-\frac{\sigma_{3}^{2}}{2}\right) \omega_{3} u_{3}^{2}+\left(\omega_{2} \beta(1-p) \frac{S^{*}}{N}+r_{2}\right)+\omega_{3} k\left(1-r_{1}\right)\right) u_{2} u_{3} \\
& +\beta(1-p) \frac{I^{*}}{N} \omega_{2} u_{1} u_{2}
\end{aligned}
$$

If we choose in (7) $\omega_{1}=\mathrm{p} \omega_{3}$ and in the fact that $u_{i}^{2}$ is locally Lipchitz function, we obtain the following inequality:

$$
\begin{aligned}
& L V \leq-\left(\beta \frac{I^{*}}{N}+\mu-\frac{\sigma_{1}^{2}}{2}\right) \omega_{1} u_{1}^{2}-\beta \frac{S^{*}}{N}\left(\omega_{1}-p \omega_{3}\right) u_{1} u_{3}-\left(\mu+k\left(1-r_{1}\right)-\frac{\sigma_{2}^{2}}{2}\right) \omega_{2} u_{2}^{2} \\
& -\left(\left(\mu+d+\delta+r_{2}\right)-\beta p \frac{S^{*}}{N}-\frac{\sigma_{3}^{2}}{2} \omega_{3}\right) u_{3}^{2}
\end{aligned}
$$

By theorem (6) the proof is complete.

\section{Computation Solution OF Stochastic Differentiable EQUATION}

Now we take account of stochastic method of E.J.Allen and al. for evaluating the transition probability density for the process which is the solution of one stochastic differential equation. To form the SDE model, we will calculate $\mathrm{E}(\Delta \mathrm{X})$ and $\mathrm{E}((\Delta \mathrm{X})(\Delta \mathrm{X}))$ The evolution of population is according to the rates on the following table:

Table1. Compartment changes in a small time period $\Delta t$

\begin{tabular}{|l|c|c||}
\hline Transition & component process & Probabilities \\
\hline $\mathrm{S} \rightarrow \mathrm{S}+1$ & {$[1,0,0]$} & $\Lambda \Delta \mathrm{t}$ \\
\hline $\mathrm{S} \rightarrow \mathrm{S}-1$ & {$[-1,0,0]$} & $\mu S \Delta \mathrm{t}$ \\
\hline $\mathrm{S} \rightarrow \mathrm{S}-1$ & {$[-1,1,0]$} & $\beta(1-p) p \frac{S I}{N} \Delta \mathrm{t}$ \\
\hline $\mathrm{S} \rightarrow \mathrm{S}-1$ & {$[-1,0,1]$} & $\beta p \frac{S I}{N} \Delta \mathrm{t}$ \\
\hline $\mathrm{E} \rightarrow \mathrm{E}-1$ & {$[0,1,-1]$} & $r_{2} I \Delta \mathrm{t}$ \\
\hline $\mathrm{E} \rightarrow \mathrm{E}-1$ & {$[0,-1,0]$} & $\mu E \Delta \mathrm{t}$ \\
\hline $\mathrm{E} \rightarrow \mathrm{E}-1$ & {$[0,-1,1]$} & $k\left(1-r_{1}\right) \mathrm{E} \Delta \mathrm{t}$ \\
\hline $\mathrm{I} \rightarrow \mathrm{I}-1$ & {$[0,0,-1]$} & $\left(\mu+d+\delta+r_{2}\right) I \Delta \mathrm{t}$ \\
\hline
\end{tabular}

The mean of system is given by

$$
E(X)=\left(\begin{array}{l}
\Lambda-\beta \frac{S I}{N}-\mu S \\
\beta(1-p) \frac{S I}{N}+r_{2} I-\left(\mu+k\left(1-r_{1}\right)\right) E \\
\beta p \frac{S I}{N}+k\left(1-r_{1}\right) E-\left(\mu+d+\delta+r_{2}\right) I
\end{array}\right) \Delta t .
$$

and the comatrix is given by:

$$
E(X(t) X(t))=\left(\begin{array}{lll}
\Lambda+\beta \frac{S I}{N}+\mu S & \beta(1-p) \frac{S I}{N} & 0 \\
-\beta(1-p) \frac{S I}{N} & \beta(1-p) \frac{S I}{N}+r_{2} I+\mu E & -k\left(1-r_{1}\right) E-r_{2} I \\
-\beta p \frac{S I}{N} & -k\left(1-r_{1}\right) E-r_{2} I & \left(\mu+d+\delta+r_{2}\right) I
\end{array}\right) \Delta t=G \Delta t .
$$


It has been proved in that $\mathrm{X}(\mathrm{t})$ is normally distributed. Then,

$$
X(t+\Delta t)+E(X(t)) \Delta t+\sqrt{G \Delta t} \gamma
$$

where $\gamma \in N(0,1)$, for $\mathrm{i}=1, . .3$

and when $\Delta t \rightarrow 0, \mathrm{X}(\mathrm{t})$ converges strongly to the solution of stochastic system :

$$
\frac{d X(t)}{d t}=E(X(t))+\sqrt{G \Delta t} \frac{d W(t)}{d t}
$$

and the next step is to calculate $\sqrt{G}$ in the form $\mathrm{G}=H H^{T}$. One will uses Allen and al. technique $[3,4]$ based on the calculate of $\mathrm{H}=\mathrm{P} D^{1 / 2} P^{T}$, if $\mathrm{G}$ is a symmetric positive definite matrix. In our model $\mathrm{G}$ is a $3 \mathrm{X} 7$ matrix , not symmetric. Hence we will use the equivalent stochastic differential equation as follow:

\subsection{Equivalent SDE}

The system (5) will be changed into SDEs in the form :

$$
d X(t)=F(t, X(t) W(t)+G(t, X(t)) d W(t)
$$

$\mathrm{F}(\mathrm{t}, \mathrm{X}(\mathrm{t}))$ is called the drift function or deterministic, $\mathrm{G}(\mathrm{t}, \mathrm{X}(\mathrm{t}))=\left(\lambda_{j, i} P_{j}^{1 / 2}\right)$ for $\mathrm{i}=1, . ., \mathrm{m} \mathrm{j}=1, . \mathrm{d}$ is called dispersion function or diffusion and $\mathrm{dW}(\mathrm{t})$ is the Brownian noise.

Using the second modeling procedure of Allen and Al.[4] we get an equivalent to the preceding model as follow:

$$
\begin{aligned}
& \left\{\begin{array}{l}
d X(t)=f(X(t)) d t+H(t, X(t)) d W(t) \\
X(0)=\left[X_{1}(0), X_{2}(0), X_{3}(0)\right]
\end{array}\right. \\
& G=\left(\begin{array}{llllllll}
\Lambda & \mu & -\beta(1-p) \frac{S I}{N} & -\beta p \frac{S I}{N} & 0 & 0 & 0 & 0 \\
0 & 0 & \beta(1-p) \frac{S I}{N} & 0 & r_{2} I & +\mu E & -k\left(1-r_{1}\right) E & 0 \\
0 & 0 & 0 & \beta p \frac{S I}{N} & -r_{2} I & 0 & k\left(1-r_{1}\right) E & -\left(\mu+d+\delta+r_{2}\right) I
\end{array}\right) \\
& H=\left(\begin{array}{llllllll}
\sqrt{\Lambda} & \sqrt{\mu} & -\sqrt{\beta(1-p) \frac{S I}{N}} & -\sqrt{\beta p \frac{S I}{N}} & 0 & 0 & 0 & 0 \\
0 & 0 & \sqrt{\beta(1-p) \frac{S I}{N}} & 0 & \sqrt{r_{2} I} & \sqrt{\mu E} & -\sqrt{k\left(1-r_{1}\right) E} & 0 \\
0 & 0 & 0 & \sqrt{\beta p \frac{S I}{N}} & -\sqrt{r_{2} I} & 0 & \sqrt{k\left(1-r_{1}\right) E} & -\sqrt{\left(\mu+d+\delta+r_{2}\right) I}
\end{array}\right)
\end{aligned}
$$

The system becomes:

$$
\left\{\begin{array}{l}
d S=\left(\Lambda-\beta \frac{S I}{N}-\mu S\right) d t+\sqrt{\Lambda} d W_{1}(t)+\sqrt{\mu} d W_{2}(t)-\sqrt{\beta(1-p) \frac{S I}{N}} d W_{3}(t)-\sqrt{\beta p \frac{S I}{N}} d W_{4}(t) \\
d E=\left(\beta(1-p) \frac{S I}{N}+r_{2} I-\left(\mu+k\left(1-r_{1}\right)\right) E\right) d t+\sqrt{\beta(1-p) \frac{S I}{N}} d W_{3}(t)+\sqrt{r_{2} I} d W_{5}(t) \\
+\sqrt{\mu E} d W_{6}(t)-\sqrt{k\left(1-r_{1}\right) E} d W_{7}(t) \\
d I=\left(\beta p \frac{S I}{N}+k\left(1-r_{1}\right) E-\left(\mu+d+\delta+r_{2}\right) I\right) d t+\sqrt{\beta p \frac{S I}{N}} d W_{4}(t)-\sqrt{r_{2} I} d W_{5}(t) \\
+\sqrt{k\left(1-r_{1}\right) E} d W_{6}(t)-\sqrt{\left(\mu+d+\delta+r_{2}\right) I} d W_{7}(t)
\end{array}\right.
$$

\subsection{Computational method and results}

In this section, computational results are given for the stochastic system. We use the EulerMaruyama method mentioned in Higham. 


$$
\left\{\begin{array}{l}
S_{k+1}=S_{k}+h\left(\Lambda-\beta \frac{S I}{N}-\mu S\right) \Delta t+\sqrt{h}\left(\sqrt{\Lambda} \eta_{1 k}(t)+\sqrt{\mu} \eta_{2 k}(t)-\sqrt{\beta(1-p) \frac{S I}{N}} \eta_{3 k}(t)-\sqrt{\beta p \frac{S I}{N}} \eta_{4 k}(t)\right) \\
E_{k+1}=E_{k}+h\left(\beta(1-p) \frac{S I}{N}+r_{2} I-\left(\mu+k\left(1-r_{1}\right)\right) E\right)+\sqrt{h}\left(\sqrt{\beta(1-p) \frac{S I}{N}} \sqrt{\eta_{3 k}}(t)+\sqrt{r_{2} I} \sqrt{\eta_{5 k}}(t)\right. \\
+\sqrt{\mu E} \sqrt{\eta_{6 k}}(t)-\sqrt{k\left(1-r_{1}\right) E} \sqrt{\left.\eta_{7 k}(t)\right)} \\
I_{k+1}=I_{k}+h\left(\beta p \frac{S I}{N}+k\left(1-r_{1}\right) E-\left(\mu+d+\delta+r_{2}\right) I\right)+\sqrt{h}\left(\sqrt{\beta p \frac{S I}{N}} \eta_{4 k}(t)-\sqrt{r_{2} I} \eta_{5 k}(t)\right. \\
\left.+\sqrt{k\left(1-r_{1}\right) E} \eta_{7 k}(t)-\sqrt{\left(\mu+d+\delta+r_{2}\right) I} \eta_{8 k}(t)\right)
\end{array}\right.
$$

The following parameters are taken from [5]: $\mu=0.101, \mathrm{r}_{1}=0.01, \delta=0.16288$,

$\mathrm{r}_{2}=0.81862, \mathrm{~d}=0.0022727 \mathrm{p}=0.1, \mathrm{~S}(0)=1000, \mathrm{E}(0)=200$ and $\mathrm{I}(0)=100$.

$\eta_{\mathrm{k}}$ are the gaussian random variables $\mathrm{N}(0,1)$.

The simulation results are depicted in following figures:

\section{Example 1:}

For $\beta=2, \mathrm{k}=0.005$ we obtain $\mathrm{R}_{0}=0.27387<1$ and the following figures 1 and 2 show that the desease will become extinct.

Table1. Mean and standard deviation for ODE epidemic model and SDE epidemic Model at $t=516$

\begin{tabular}{|l|cc|c|}
\hline Models & Variables $\left(\mathrm{X}_{\mathbf{i}}\right)$ & $\mathrm{E}(\mathrm{X})$ & $\sigma(\mathrm{X})$ \\
\hline \multirow{3}{*}{ Ode } & $\mathrm{S}$ & 1075 & 0 \\
& $\mathrm{E}$ & 337 & 0 \\
& $\mathrm{I}$ & 2 & 0 \\
\hline \multirow{3}{*}{$\mathrm{SDE}$} & $\mathrm{S}$ & 796 & 80,36 \\
& $\mathrm{E}$ & 89 & 41,76 \\
& $\mathrm{I}$ & 1 & 8,95 \\
\hline
\end{tabular}

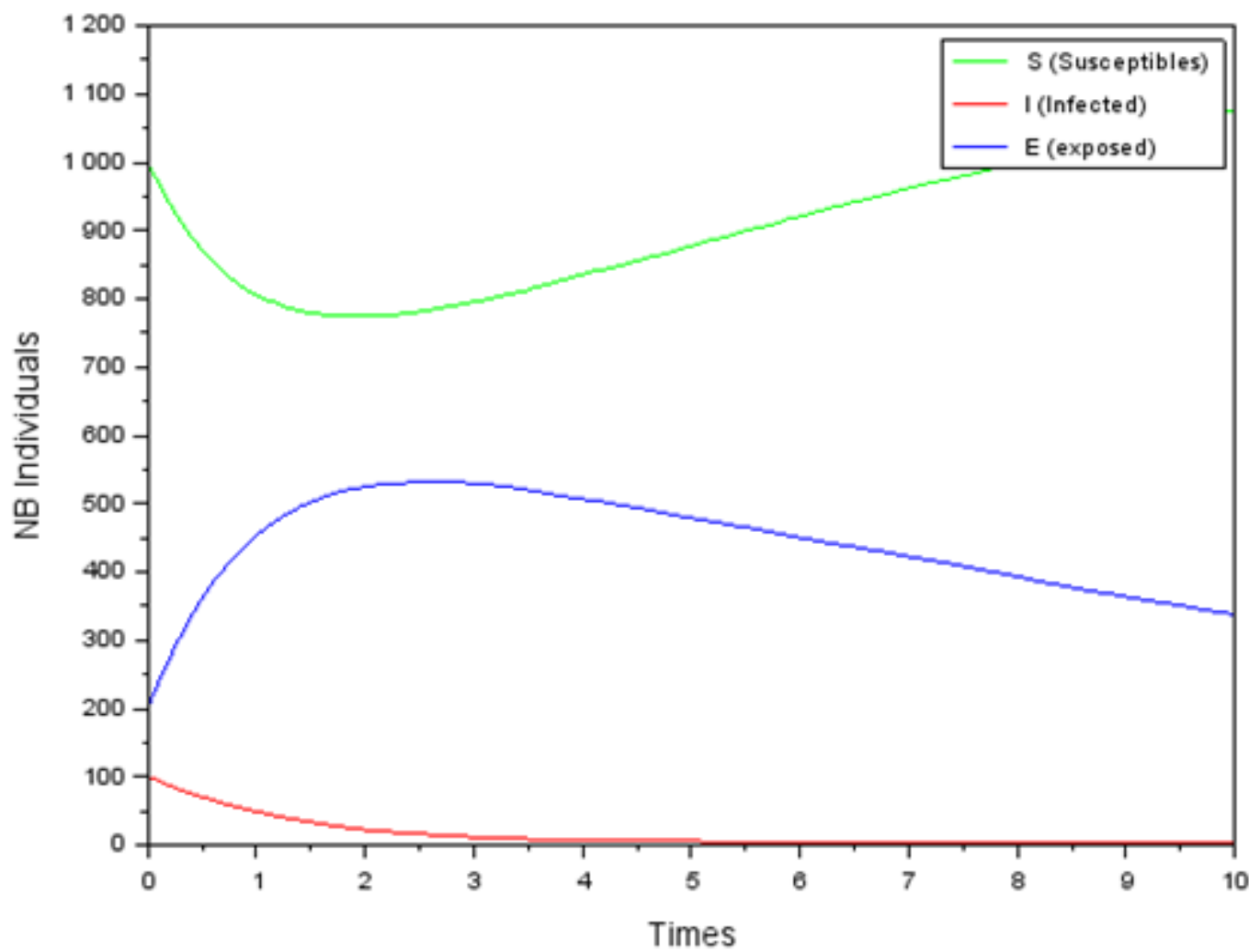

Fig.1. represents the solution of dynamical model with $R_{O}<1$ 


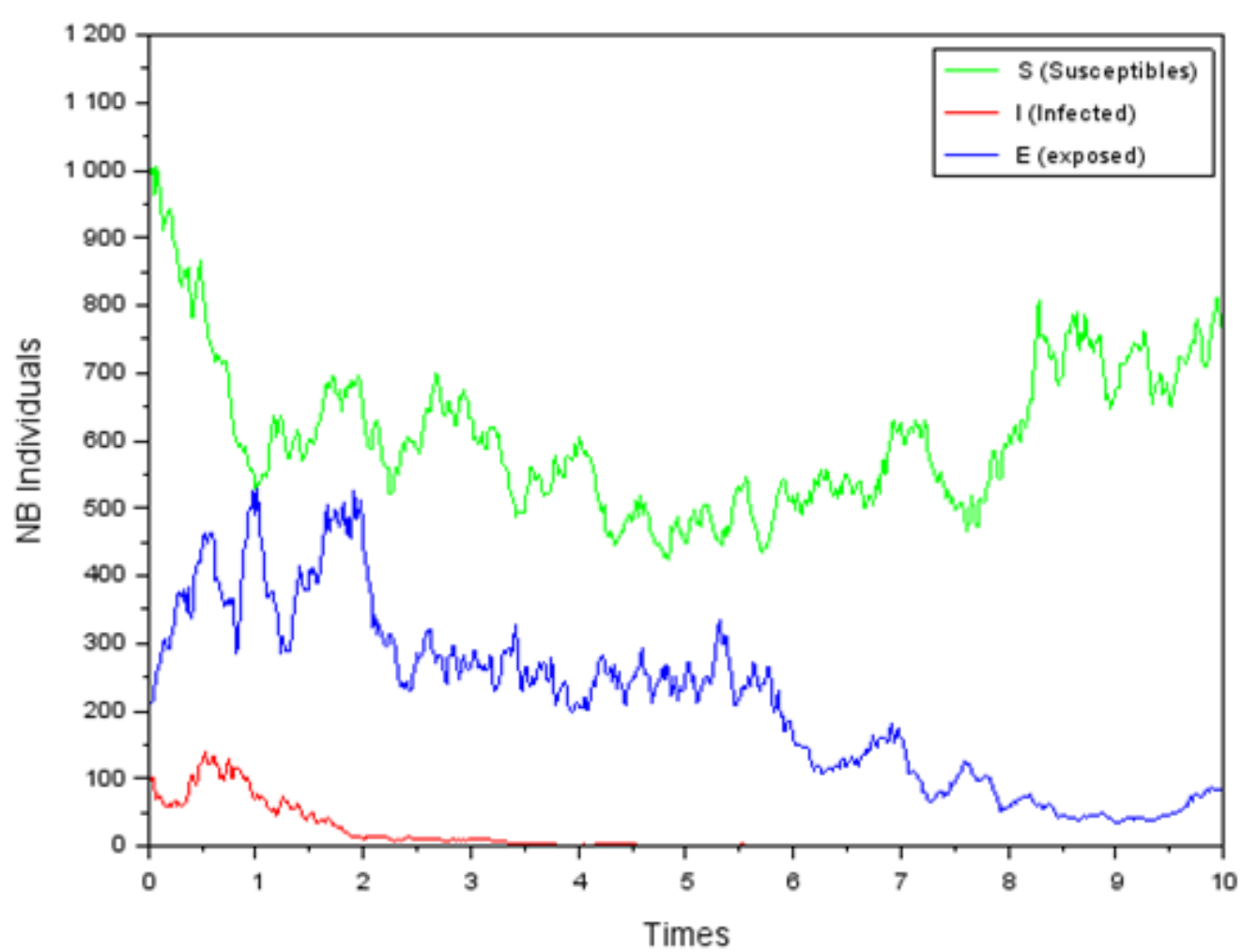

Example 2:

Fig 2. represents the solution of stochastic model with $R_{0}<1$

For $\beta=20$, we have $\mathrm{R}_{0}=2.7387$ and on figures 3 and 4 , the desease will persist.

Table 2. Mean and standard deviation for ODE epidemic model and SDE epidemic model at $t=516$, for $R_{0}=2.7387$

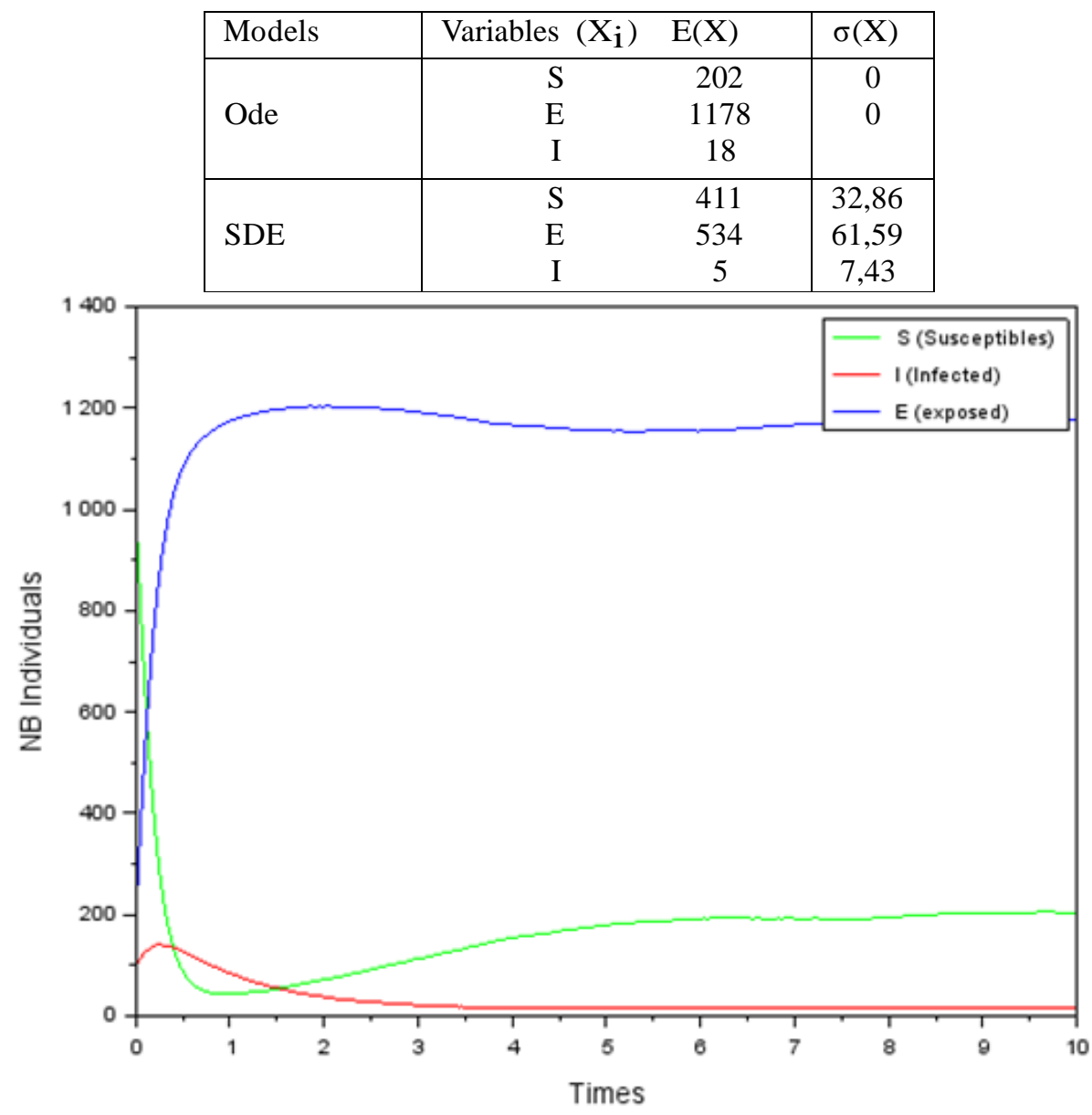

Fig 3 represents the solution of stochastic model with $R_{0}>1$ 


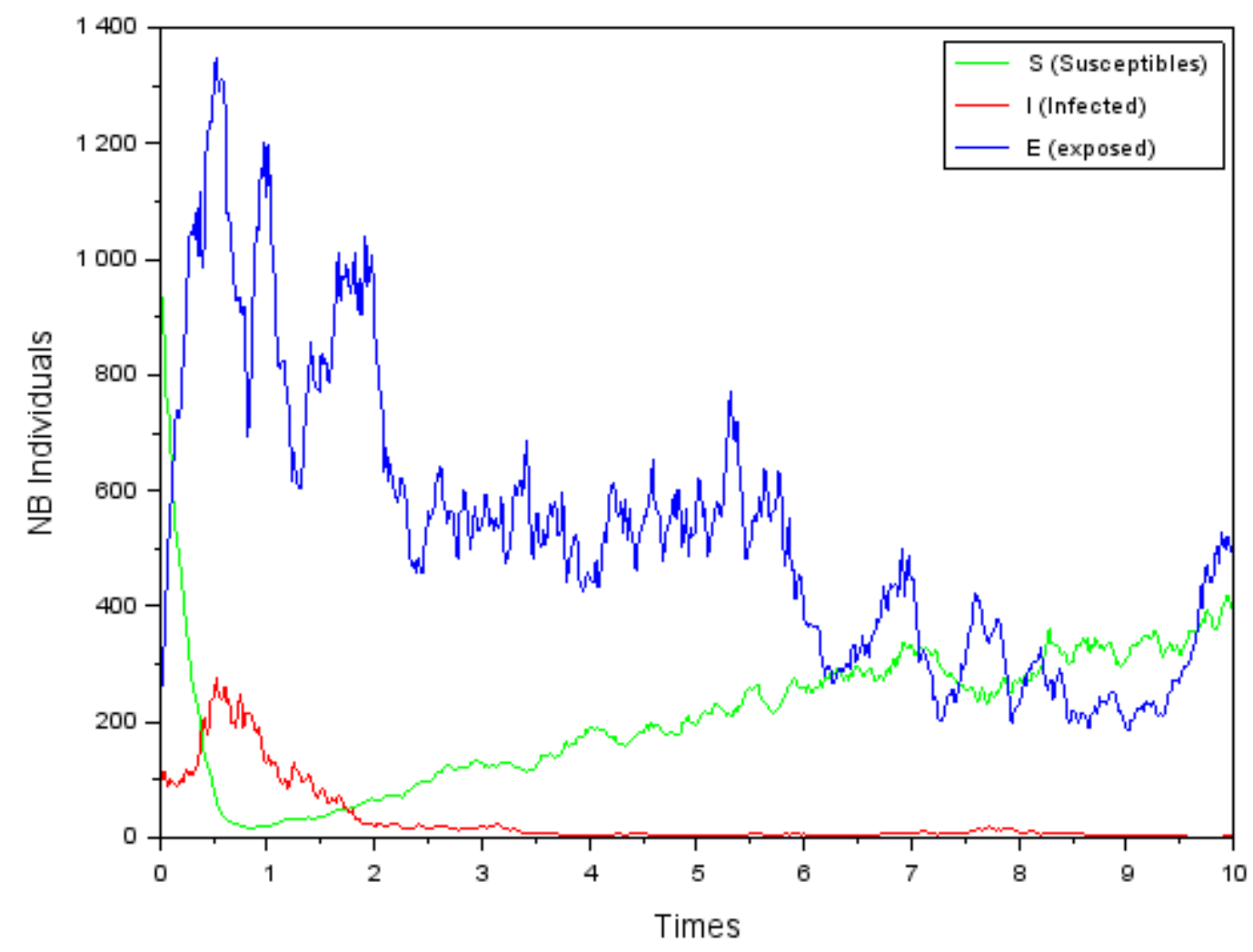

Fig 4. represents the solution of stochastic model with $R_{0}>1$

\section{CONCLUSION}

In this paper, we have considered a stochastic model tuberculosis model. The sta- bility of the positive equilibium and the existence are investigated by Lyapounov func- tions.Deterministic and equivalent stochastic models are presented. Finally some numeri- cal simulations are also included to testify the validity of the theoretical results. It appear that deterministic and stochastic models have the same trend.

\section{REFERENCES}

[1]. V. N. Afanasev, V. B. Kolmanowski and V.R. Nosov Mathematical Theory of Global Systems Design, Kluwer, Dordrecht, 1996

[2]. J Arino, C. McCluskey and P. Van den Driessche Global results for an endemic model with vaccination that exhibits backward bifurcation Siam Journ. on applied Math., 64, 2003, 260-276

[3]. E.J. Allen, J. Baglama, S.K. Boyd:Numerical approximation of the product of the square root of matrix with vector Linear and its applications , Vol. 26, No. 2, 2008,pp 274-281

[4]. E.J.Allen, L.J. Allen, A. Arciniega and P.E. Greenwood Construction of an equivalent stochastic differential equ tion models Stochastic analysis and applica- tions, Vol. 26,n2, 2008,pp 274-297

[5]. Bowong S. and Tewa J.J.Gobal analysis of a dynamical model for transmission of tuberculosis with general contact rate comm. in nonlinear sciences and numerical simulations pp3621-3631, 2010

[6]. Priscillia E. Greenwood and Luis F. Gordillo Stochastic epidemic modeling (2006)

[7]. D. J. HighamAn algorithm introduction to numerical simulation of stochastic diffe- rential equations SIAM review vol. 43, N3, pp525-546, 2001

[8]. J.P. LaSalle The stability of.dynamical systems., Socierty for Industrial and Applied Mathematics Vol. 25(1987). 
[9]. H. El Maroufy, A. Lahrouz, PGL Leach :Qualitative behaviour of a model of sirs epidemic : stability and permanence Applied Mathematics \& information sciences vol. 5, No 2, pp 220-238 2011

[10].S. Ouaro, A. Traoré: Detereministic and stochastic schistosomiasis model with general incidence, Applied Mathematics 2013, 4, 1682-1693

[11].P. Van den Driessche, J. Watmough Reproduction numbers and sub-thresolds endemic equilibria for compartimental models of diseases transmission Math. Bios- ciences vol. 180, pp29-48

[12].D. Mukherjee : Stability Analysis of a Stochastic Model for Prey-Predator System with Disease in the Prey, Nonlinear Analysis: Modelling and Control, 2003, Vol. 8, No. 2, 83-92 